\title{
Comparative Wound Healing Effects of Honey, Olive Oil, Pawpaw Fruit Extract and Iodine in Diabetic Rats: an Evaluation and Prioritization of Potential Alternative Therapeutic Options
}

\author{
Magnus Michael Chukwudike Anyakudo* and Oluwabukola Mary Ayibiowu \\ Endocrinology/Metabolism and Clinical Nutrition Research Unit of Department of Physiology, University of Medical Sciences, Nigeria
}

*Corresponding author: Magnus Michael Chukwudike Anyakudo, Endocrinology/Metabolism and Clinical Nutrition Research Unit of Department of Physiology, Faculty of Basic Medical Sciences, University of Medical Sciences, P.M.B 536, Ondo City, Ondo State, Nigeria

\begin{abstract}
High cost of conventional diabetic wound management is of public interest due to their social and economic burden on the individuals and care givers. This experimentally-controlled designed study compared the wound healing potentials of natural products: Honey, Olive oil, Pawpaw fruit (unripe mesocarp) extract (PFE) and Iodine (positive control) in diabetic rats. Twenty-eight male Wistar rats categorized into four groups $(n=7$, each) weighing between $150-200 \mathrm{~g}$ were used for this study which lasted four weeks. A full thickness excision wound of circular area $300 \mathrm{~mm} 2$ and $2 \mathrm{~mm}$ in-depth was inflicted on all rats after light anesthesia with i.v. ketamine ( $90 \mathrm{mg} / \mathrm{kg}$ b.w.). Aliquot portions of Olive oil, Honey, Iodine and PFE were topically applied to the opened wounds twice a day. Wound areas were measured on days 1, 7, 14, 21 and 28 using a transparent sheet and a permanent marker. Wound healing activity was assessed using percentage area of wound contraction, epithelization period and granulation tissue integrity. Data were analyzed using Microsoft Excel and SPSS v. 22. Comparison between groups was made using one way ANOVA. P values $<0.05$ were considered significant. Significant wound contraction occurred on $14^{\text {th }}$ day of treatment in all groups: Iodine (99.4\%), Olive oil (98.4\%), Honey (92.4\%) and PFE (90.2\%) with different remarkable epithelization periods (days): Iodine (14), Olive oil (15), Honey (16) and Pawpaw extract (17). Granulation tissue integrity improved respectively in the order of PFE, Honey, Olive oil and Iodine. The results of this study revealed that honey, olive oil and PFE as natural products have varying beneficial effects on wound healing process in diabetic rats.
\end{abstract}

Keywords: Natural Products; Wound Healing; Diabetic Rats; Alternative Therapeutic Options

\section{Introduction}

Wound healing is an intricate and dynamic process of replacing devitalized and missing cellular structures and tissue layers following injury [1,2]. This complex process involves phases of hemostasis, inflammation, proliferation and remodeling [3]. Though, the exact pathogenesis of poor and delayed wound healing in diabetics is not clearly understood, evidences from human and animal studies however, have revealed several abnormalities in the various phases of wound healing process. Individuals with diabetes demonstrate reduced capability in acute wound healing associated with susceptibility to chronic diabetic foot ulcer development, a serious complication reported to affect $15 \%$ of people with diabetes and accounts for $85 \%$ of all diabetes-related lower leg amputations [4]. This impaired healing abilities of diabetics result from multiple pathophysiological mechanisms involving hypoxia, fibroblast and epidermal cell dysfunction, impaired angiogenesis and neovascularization, high levels of metalloproteases, damage from reactive oxygen species and AGEs (advanced glycation endproducts), decreased host immune resistance and neuropathy [5]. Delayed wound healing and chronic wound management in diabetics is of public interest due to their social and economic burden on the individuals and care givers. To abate such burden, a paradigm shift to beneficial alternative therapy which is affordable, accessible, available and competitively effective compared to conventional wound management, is recently attracting focus. This study therefore, compared the wound healing effects of iodine, honey, olive oil and pawpaw fruit extract (locally available, accessible and affordable in our environment) in experimental diabetic rats with rationale to evaluate and prioritize their therapeutic potentials as alternative remedies in wound healing. 


\section{Methodology}

The unadulterated original Honey, Olive oil, Iodine and Pawpaw (unripe) used in this study were identified and purchased at a local market in western part of Nigeria with the assistance of an agriculturist and a chemist. The pawpaw (Carica papaya) fruit extract was prepared by washing and properly cleaning the fruit with distilled water while the outer green thin layer (exocarp) was peeled and discarded to expose the underlying mesocarp which was cut and weighed. $200 \mathrm{~g}$ of the peeled mesocarp was blend with $50 \mathrm{ml}$ of distilled water to a fine texture form using a laboratory blender. The mixture obtained was filtered using a fine muslin cloth to produce a residue that was oven dried at $40^{\circ} \mathrm{C}$ to a white colored powdery form. The residue was weighed and stored in waterproof container

Twenty-eight (28) male albino Wistar rats (Rattus norvegicus) weighing about 150 - 200g used for this study were obtained from the disease-free stock of Olu farm, Ibadan, Oyo State, Nigeria. The animals were kept in polypropylene cages with stainless wire mesh top in a well-ventilated animal house maintained at normal and standard laboratory conditions of temperature and relative humidity with access to standard rat pellets and water ad libitum for the entire experimental period. Animal Ethical Committee of the institution approved the study protocol. After 15 hour overnight fast following acclimatization, all rats were injected by single intraperitoneal injection of $150 \mathrm{mg} / \mathrm{kg}$ body weight of freshly prepared 2\% Alloxan monohydrate (Sigma chemicals, USA) dissolved in sterile $0.9 \%$ normal saline in a standard volumetric flask strapped with foil to prevent alloxan instability. Diabetes was confirmed 4-7 days later by use of commercial glucometer (On Call Plus Blood Glucose Monitoring System, ACON Laboratories, Inc. San Diego, USA.) and compatible strips. Rats with Fasting Blood Glucose (FBG) level $>150 \mathrm{mg} / \mathrm{dl}$ were considered diabetic and used for this study since the level of serum glucose considered to be normal in Rattus norvegicus ranges from 50 - $135 \mathrm{mg} / \mathrm{dL}$ [6]. Diabetes was allowed to stabilize for 5 days before animal grouping and wound infliction. Fasting blood glucose levels of all rats in each experimental group were measured on weekly basis for the four week study period. After diabetes induction, the animals were categorized into four groups of seven rats each. Thereafter, the rats were inflicted with excision wounds treated according to the experimental design as shown below:

Group A: Diabetic rats with wounds treated with Iodine (Positive control)

Group B: Diabetic rats with wounds treated with Honey

Group C: Diabetic rats with wounds treated with Olive oil
Group D: Diabetic rats with wounds treated with Pawpaw fruit extract

A modified method of Anyakudo and Erinfolami [7] was used to excise wound in the rats. Animals were anaesthetized with $0.48 \mathrm{ml}$ of intravenous ketamine hydrochloride $(90 \mathrm{mg} / \mathrm{kg}$ body weight) and shaved on the dorsolateral side of the thoracic region using scissors. The area of the wound to be created was outlined on the shaved part of the animals with a blue ink marker while a full thickness excision wound of circular area $300 \mathrm{~mm}^{2}$ and 2 $\mathrm{mm}$ in depth was created along the markings after disinfected with $70 \%$ ethanol. Animals were allowed to stabilize for $24 \mathrm{hrs}$ from the surgery. Thereafter, the treatment (test) materials were topically applied to the wounds without covering with any wool or plaster. Animals were carefully observed and cages kept clean to prevent infection. The treatment materials were applied topically twice a day. Wound areas were measured on days 1, 7, 14, 21 and 28 using a transparent sheet and a permanent marker. Wound healing activity was assessed using percentage area of wound contraction, epithelization period and granulation tissue integrity. Data were analyzed using Microsoft excel and SPSS v. 22. Results were expressed as mean \pm SEM. Comparison between groups was made using one-way ANOVA while $\mathrm{P}$ values $<0.05$ were considered significant.

\section{Results}

The profile of wound healing contraction and epithelization in various groups is shown in Table 1 and 2 below. On 14th day of the study, a comparable significant $(p<0.05)$ increase in the area of wound contraction was observed within all groups: Iodine (99.4\%), Olive oil (98.4\%), Honey (92.4\%) and Pawpaw extract (90.2\%) while on the 28th day, all wounds were observed to be completely healed. Difference in areas of wound contraction when compared across groups was also significant. Attainable period (expressed in days) for observed remarkable epithelization during wound healing differs with treatment option: Iodine (14), Olive oil (15), Honey (16) and Pawpaw extract - PFE (17). These results reflected their differential healing rate potentials. Also, well-organized granulation tissues were formed during the healing process in all groups. However, granulation tissue integrity was remarkably highest in iodine-treated group followed by olive oil, honey and PFE respectively. Plate A shows the typical representation of all grouped diabetic rats on Day 1 after wound infliction while Plate B1- 4 and C1- 4 depict the wound healing processes of PFE and honey respectively on Days 7, 14, 21 and 28. The honey and pawpaw fruit extract pictures were chosen for display here because they are readily available and accessible to a common individual in our environment. The iodine in this study serves as a positive control (Figure 1). 


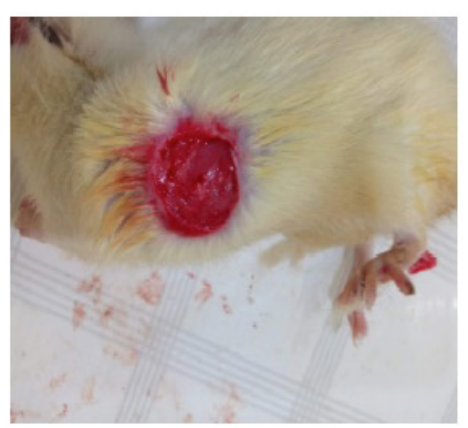

PLATE A: ANAESTHETIZED RAT INFLICTED WITH WOUND ON DAY 1

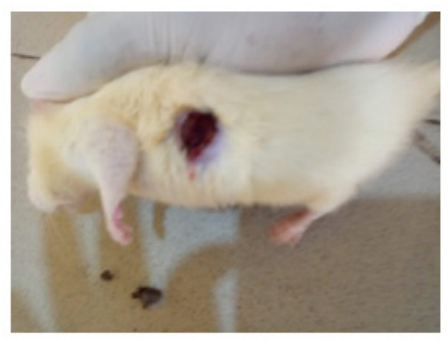

PFE DAY 7

PLATE B1

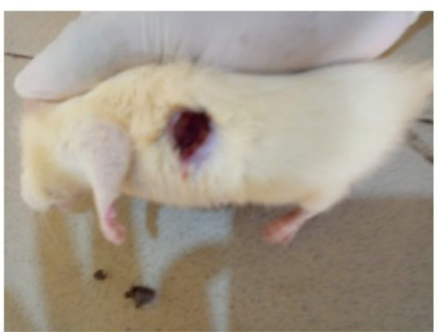

HONEY DAY 7

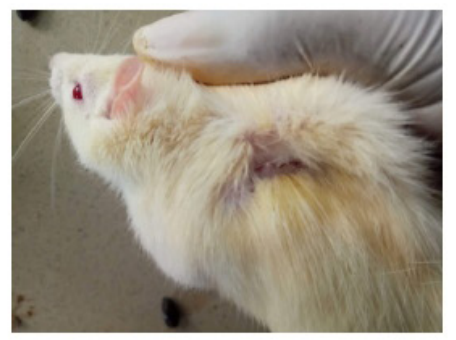

PFE DAY 14

PLATE B2

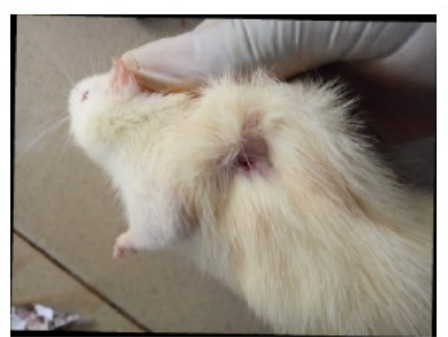

HONEY DAY 14

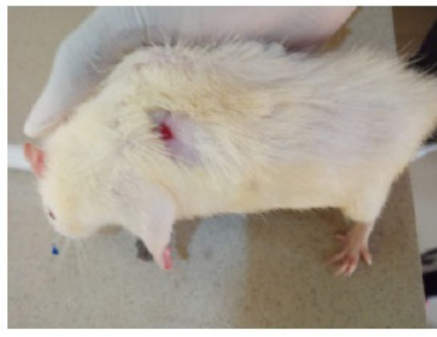

PFE DAY 21

PLATE B3

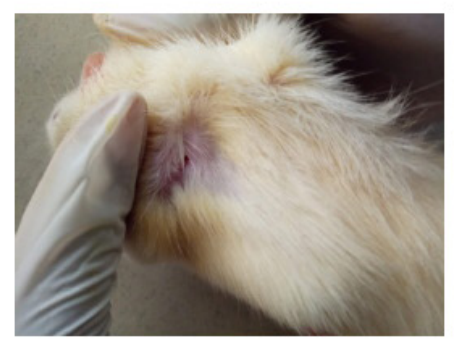

HONEY DAY 21

PLATE C3

\section{PLATE C1}

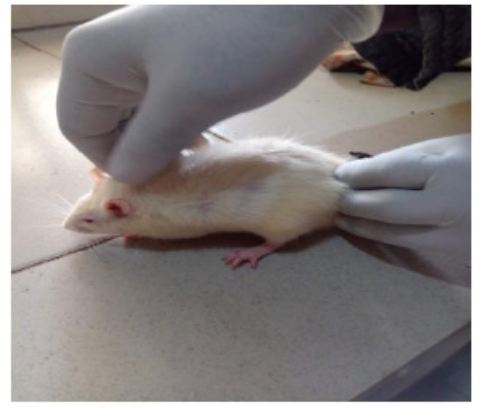

HONEY DAY 28
PLATE C2

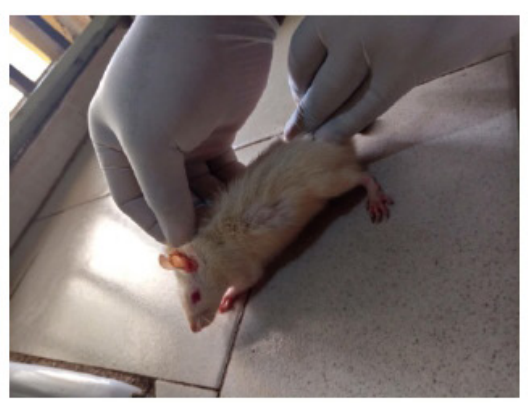

PFE DAY 28

PLATE B4

Figure 1. 


\section{Discussion}

This experimentally controlled designed study which lasted four weeks compared the wound healing activities of Honey, Pawpaw fruit extract, Olive oil and Iodine in grouped experimental diabetic rats. In this study, the different treatment options comparatively investigated caused significant different effects on area of wound contraction, degree of epithelization and granulation tissue formation used to assess their potentiality and benefit in wound healing. Wound healing activities was remarkably highest in iodinetreated group (positive control) followed by olive oil, honey and PFE respectively (Tables 1,2). The observed therapeutic effect of pawpaw fruit extract in this study on wound healing in diabetic rats correlates with the finding of previous study [7] which proved the alternative benefit of aqueous mesocarp extract of pawpaw fruit in wound treatment in healthy and diabetic individuals. Topical use of Carica papaya fruit in chronic ulcers treatment was considered to be more effective than other topical applications according to Hewitt et al [8]. However, in this study, Olive oil and Honey showed better improvement on wound healing process over that of papaya. Different parts (fruit, leaf, stem, latex, root) of this tropical tree called Carica papaya Linn (family: Caricacaea) with over 1000 species worldwide have long been reported and recently reviewed to be useful as herbal medicines or concoctions in the management of various disorders including diabetes and wound infection [9, 10]. Carica papaya various parts are variably rich in Vitamin A and $\mathrm{C}$ and other several biochemical compounds that may explain the mechanism of its beneficial impact on wound healing process [11]. Pawpaw is a common fruit in various human habitats especially in the tropic and subtropic areas. Therefore, planting of pawpaw should be encouraged because of its various health benefits including wound treatment.

Table 1: Wound healing activities of treatment materials in grouped diabetic rats ( $n=5 /$ group).

\begin{tabular}{|c|c|c|c|c|}
\hline \multirow{2}{*}{ Periods } & \multicolumn{4}{|c|}{ Wound Contraction Area (mm2) in Grouped Diabetic Rats } \\
\cline { 2 - 5 } & Honey & PFE & Iodine & Olive oil \\
\hline Day 1 & $314.15 \pm 0.03$ & $314.15 \pm 0.03$ & $12.15 \pm 0.03$ & $15.15 \pm 0.03$ \\
\hline Day 7 & $176.72 \pm 0.08$ & $261.59 \pm 0.06$ & $1.77 \pm 0.03$ & $4.91 \pm 0.07$ \\
\hline Day 14 & $23.76 \pm 0.03$ & $30.70 \pm 0.06$ & $0.2 \pm 0.03$ & $1.77 \pm 0.0$ \\
\hline Day 21 & $3.14 \pm 0.03$ & $4.91 \pm 0.03$ & 0 & 0 \\
\hline Day 28 & 0 & 0 & 0.18 & \\
\hline
\end{tabular}

Values are expressed in mean \pm SEM

Table 2: Period of observed remarkable epithelization in grouped diabetic rats $(n=5 /$ group).

\begin{tabular}{|c|c|c|c|c|}
\hline \multirow{2}{*}{} & \multicolumn{4}{|c|}{ Experimental Groups } \\
\cline { 2 - 5 } & Honey & PFE & Iodine & Olive oil \\
\hline $\begin{array}{c}\text { Period of observed remarkable Epithelization } \\
\text { (Days) }\end{array}$ & $16.1 \pm 0.2$ & $17.2 \pm 0.16$ & $14.0 \pm 00$ & $15.0 \pm 0.0$ \\
\hline
\end{tabular}

Values are expressed in mean \pm SEM

The use of honey as a nutraceutical has become of increasing interestin the recentyears largely due to an increase in theavailability of evidence-based findings demonstrating the health beneficial effects of honey in treating diverse disease conditions including diabetes mellitus. In this study, honey showed a remarkable wound healing activities better than PFE but less effective compared to olive oil based on the criteria used for assessing wound activities in this study. Previous studies [12,13] carried on human subjects with diabetic foot ulcers revealed that use of honey remarkably improved wound healing process and reduce associated pain and cost of healthcare. These findings are similar to that observed in this study using animal model. The healing properties of honey based on various research studies can be attributed to its antimicrobial and anti-inflammatory effects, wound bed moisturizing, osmotic effects, decreasing edema in the cells of the wound, accelerating the process of angiogenesis and granulation in the wound, accelerating collagenases and epithelialization in the wound, increasing activities of lymphocytes and phagocytes and accelerating debridement of necrotic tissue $[14,15]$. Based on the above evidences, use of honey as a treatment option in wound healing should be given high consideration especially in diabetic wounds for expected maximum outcome. However, precautions should be taken to avoid the use of adulterated honey sold in the market which can complicate wound healing process.

Olive oil and Iodine demonstrated excellent wound healing effects in this study as revealed by their shorter periods of epithelization, faster rate of healing process and very high percentage area of wound contractions compared with honey and PFE. Application of oil in wound treatment has long been reckoned with human existence. Though, different types of oil exist in nature, preference for olive oil by man for various uses including spiritual have been established. In our environment, olive oil is almost found in every home with poor knowledge of its use in wound management. This study therefore proved the efficacy of olive oil in the treatment of wounds with beneficial outcome as also agreed by findings of other studies $[16,17]$ which revealed the remarkable wound healing effect of olive oil in the management of diabetic foot ulcers. Therefore, olive oil use in diabetic wound treatment should be highly encouraged and considered. Though the mechanism of wound healing effect of olive oil is inexplicit, It appears that it achieves its wound healing effect through mechanisms that stimulate cell healing and acceleration of wound healing process 
possibly by its constituent essential fatty acids such as linoleic acid and linolenic acid and improve granulation tissue formation by its antioxidant, antimicrobial, and anti-inflammatory properties [18]. Iodine use in the treatment of wound has long gained recognition in various homes and schools and can be seen as an orthodox or conventional therapeutic remedy for acute wound management. However, Iodine is inaccessible to most people in our environment especially those in the rural areas. Therefore, use of alternative remedies such as honey, pawpaw fruit extract and olive oil in open wounds treatment should be encouraged in place of expensive and inaccessible conventional therapies.

In future, it is hoped that the study on the effect of the combination of the above natural products may be considered to assess their synergistic or complementary effects in wound healing. Meanwhile, with the above findings, use of Olive oil, Honey and mesocarp extract of unripe pawpaw should be recommended for wound healing and be considered as alternative therapeutic options in place of expensive conventional options.

\section{Conclusion}

Diabetic wound healing has been reported generally slow if inappropriately cared for; and in the face of financial constraint as common with conventional management, this might last for weeks or even months with possibility of risk of amputation. To minimize these financial burden and associated risks based on the findings of this study, use of honey, olive oil and pawpaw fruit extract which are readily available, accessible and affordable as alternative therapeutic options should be given consideration and prioritization in our environments as recommendation for wounds care especially in diabetics.

\section{References}

1. Nguyen DT, Orgill DP, Murphy GF (2009) The Pathophysiologic Basis for Wound Healing and Cutaneous Regeneration. Biomaterials for Treating Skin Loss PP: 25-57. Orgill DP, Blanco C (Editors).

2. Thu HE, Zulfakar MH, Ng SF (2012) Alginate based bilayer hydrocolloid films as potential slow-release modern wound dressing. Int J Pharm 434 (1-2): 375-83.

3. Stadelmann WK, Digenis AG, Tobin GR (1998) Physiology and Healing Dynamics of Chronic Cutaneous Wounds. American Journal of Surgery. 176 (2A Suppl): 26S-38S.
4. Brem H, Tomic Canic M (2007) Cellular and Molecular Basis of Wound Healing in Diabetes. The Journal of Clinical Investigation 117(5):1219 $-1222$.

5. Guo S, Dipietro LA (2010) Factors Affecting Wound Healing. Journal of Dental Research 89 (3): 219 - 229.

6. Harkness JE and Wagner JE (1993) Biology and Clinical Rabbits and Rodents. (3rd edition). Sao Paulo, Roca: 48-55

7. Anyakudo MMC and Erinfolami AB (2015) Glycemic and Wound Healing Effects of Aqueous Mesocarp Extract of Unripe Carica papaya (Linn) in Diabetic and Healthy Rats. World Journal of Nutrition and Health 3(3): 47-52.

8. Hewitt H, Whittle S, Lopez J, Bailey E and Weaver S (2002) Topical Use of Papaya in Chronic Skin Ulcer Therapy in Jamaica. West Indian Medicine Journal 49(1): 32 -33.

9. Krishna KL, Paridhavi M and Patel JA (2008) Review on Nutritional, Medicinal and Pharmacological Properties of Papaya (Carica Papaya Linn). Natural Product Radiance 7(4): 364- 373.

10. Lidiani F Santana, Aline C Inada, Bruna Larissa Spontoni do Espirito Santo, Wander FO Filiú, Arnildo Pott, et al. (2019) Nutraceutical Potential of Carica papaya in Metabolic Syndrome. Nutrients 11(7): 1608.

11. Vij T and Prashar Y (2015) A Review on Medicinal Properties of Carica papaya Linn. Asian Pac J Trop Disease 5(1): 1-6.

12. Surahio AR, Khan AA, Farooq M, Fatima I (2014) Role of honey in Wound Dressing in Diabetic Foot Ulcer. J Ayub Med Coll Abbottabad 26(3): 304306.

13. Hashim Mohamed, Mansour Abu Salma, Badriya Al Lenjawi, Seham Abdi, Zaghloul Gouda, et al. (2015) The Efficacy and Safety of Natural Honey on the Healing of Foot Ulcers: A Case Series. Wounds. 27(4): 103-114.

14. Hussain MB (2018) Role of honey in Topical and Systemic Bacterial Infections. J Altern Complement Med 24(1): 15-24.

15. Delshad E, Tavakkoli Kakhki M, Motavasselian M (2017) Successful Repair of Diabetic Foot Ulcer with Honey-Based Treatment: a case report. Iran Red Crescent Med J 19(3): e41939.

16. Nasiri M, Fayazi S, Jahani S, Yazdanpanah L, MH Haghighizadeh (2015) The Effect of Topical Olive oil on The Healing of Foot Ulcer in Patients with Type 2 Diabetes: A Double-Blind Randomized Clinical Trial Study in Iran. J Diabetes Metab Disord 14: 38.

17. Elshenawie HA, Ahmed Shalan WE, Aziza EA (2013) Effect of Ozone Olive Oil Ointment Dressing Technique on the Healing of Superficial and Deep Diabetic Foot Ulcers. Journal Am Sci 9(11): 235-250.

18. Abooei MM, Eidi A, Mortazavi P (2016) Study of Effect of Olive Oil on Reepithelialization of Epithelial Tissue in Excision Wound Healing Model in Rats. J Comp Pathophysiol 13(2): 1875-1883.

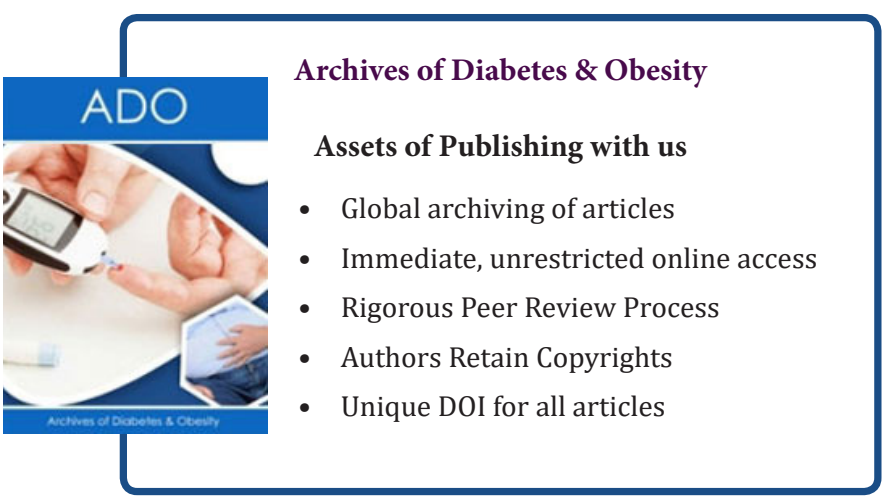

